\title{
Decrease in inflammatory response does not prevent placental dysfunction after fetal cardiac bypass in goats
}

\author{
Cheng-Bin Zhou, MD, Jian Zhuang, MD, Ji-Mei Chen, MD, Xiao-Hua Zhang, MD, and \\ Raphael C. Lui, MD
}

Objective: One of the most significant responses to fetal cardiac bypass is severe placental dysfunction characterized by increased vascular resistance. We tested the hypothesis that fetal cardiac bypass triggers the activation of nuclear factor kappa-B (NF-KB), a major regulator of inflammatory response, and that pharmacologic inhibition of NF-KB activation by pyrrolidine dithiocarbamate alleviates fetal cardiac bypass-induced placental dysfunction.

\begin{abstract}
Methods: Fifteen pregnant goats at 120 to 140 days' gestation were equally divided into the control group with a sham procedure of fetal sternotomy and cannulation (CG), the fetal bypass group (FB), and the fetal bypass group with $300 \mathrm{mg}$ pyrrolidine dithiocarbamate before sternotomy (FP). Fetal cardiac bypass was performed for 30 minutes. Umbilical arterial flow rate was measured by ultrasonic flowmeter and placental vascular resistance was calculated. Fetal plasma levels of nitric oxide (NO), endothlin-1 (ET-1), 6-keto-prostaglandin F1 $\alpha$ (6-K), thromboxane $\mathrm{B}_{2}$ (TXB2), interleukin 6 (IL-6), and tumor necrosis factor- $\alpha$ (TNF- $\alpha$ ) were assayed. IL- 6 and TNF- $\alpha$ mRNA were analyzed by real-time polymerase chain reaction. NF-KB activation was evaluated by electrophoretic mobility shift assay.
\end{abstract}

Results: Placental vascular resistance significantly increased in the FB and FP groups compared with the CG group. Increases in plasma levels of $\mathrm{NO}$ were observed in all 3 groups. Plasma levels of ET-1 rose significantly in the FB and FP groups without noticeable difference between them. Plasma levels of 6-K, TXB 2 , IL-6, and TNF- $\alpha$ increased significantly in the FB group compared with the CG and FP groups. The transcription levels of IL- 6 and TNF- $\alpha$ mRNA in the placental tissues of the FB group were significantly higher than in the FP and CG groups. The amount of activated NF-KB in the placental tissues of the FB group was also significantly higher than that in the FP and CG groups.

Conclusions: Fetal cardiac bypass-induced inflammatory response possibly mediated by NF-KB caused placental dysfunction. Pharmacologic inhibition of NF-KB activation and decrease in the inflammatory response did not alleviate the placental dysfunction. (J Thorac Cardiovasc Surg 2012;143:445-50)

It is believed that in utero correction of certain congenital heart defects may benefit the growth and development of the heart, preventing secondary changes from abnormal blood flow patterns. Fetal cardiac surgical intervention often requires extracorporeal circulation support with undesirable side effects. The most significant response to fetal extracorporeal circulation is severe placental dysfunction characterized by increased vascular resistance, reduced blood flow, and subsequent impairment of fetal gas

\footnotetext{
From the Department of Cardiovascular Surgery, Guangdong Provincial Cardiovascular Institute, Guangdong General Hospital, Guangdong Academy of Medical Sciences, Guangzhou, China.

This work was supported by grants from National Natural Science Foundation of China (30300346)

Disclosures: Authors have nothing to disclose with regard to commercial support.

Received for publication Jan 3, 2011; revisions received May 26, 2011; accepted for publication July 11, 2011; available ahead of print Aug 8, 2011.

Address for reprints: Jian Zhuang, MD, the Department of Cardiovascular Surgery, Guangdong Provincial Cardiovascular Institute, Guangdong General Hospital, 96 Dongchuan Rd, Guangzhou, Guangdong, China 510080 (E-mail: zhuangjian5413@gmail.com).

0022-5223/\$36.00

Copyright (C) 2012 by The American Association for Thoracic Surgery

doi:10.1016/j.jtcvs.2011.07.011
}

exchange. The pathophysiology of postbypass placental dysfunction includes release of prostanoids, endothelial dysfunction, leukocyte and complement activation, and other inflammatory pathways. ${ }^{1-6}$

Umbilical vessels are not innervated and therefore vasodilatation must occur through direct vasorelaxant paracrine effect of agents acting on placental vessels in their physiologic state. Several agents modulate the tone of the cord and villous vessels of the placenta, such as vasodilatation agents prostacyclin, nitric oxide (NO), atrial natriuretic peptide and vasoconstriction agents thromboxane $\mathrm{A}_{2}$ (TXA2), endothelin-1 (ET-1), and angiotensin. ${ }^{7-10}$ Most of these are proinflammatory mediators.

Nuclear factor kappa-B (NF-KB) is a major transcription factor involved in the regulation of inflammatory response. Under physiologic conditions, NF-KB combines with inhibitor kappa-B and is stored in the cytoplasm. On activation, NF-KB translocates to the nucleus and binds the sequence GGGRNNYYCC on target gene promoters, initiating transcription of genes for cyclooxygenase, nitric oxide synthase (NOS), ET-1, angiotensin, and some inflammatory mediators, such as interleukin (IL) 6 and tumor necrosis 


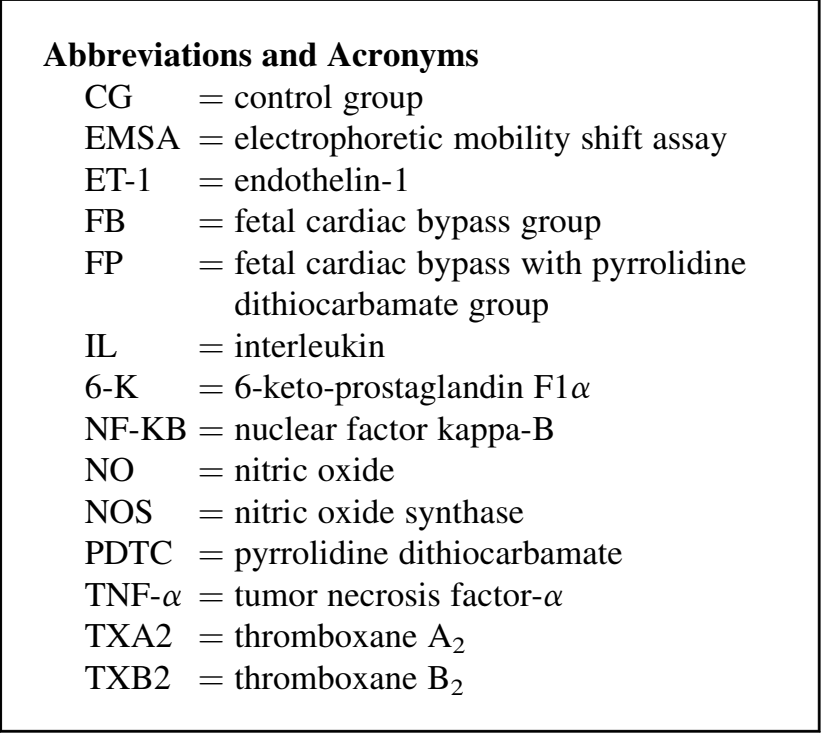

factor- $\alpha$ (TNF- $\alpha)$. NF-KB is also involved in cellular development and labor. ${ }^{11-14}$ We hypothesize that fetal cardiac bypass activates NF-KB, causing an inflammatory mediator burst that constricts placental vessels and that pharmacologic inhibition of NF-KB might alleviate the placental dysfunction induced by fetal cardiac bypass. We used fetal goat cardiac bypass as an animal model to test our hypothesis.

\section{MATERIALS AND METHODS}

The study protocol was approved by the Animal Research Committee of Guangdong General Hospital. All animals were handled in accordance with the Guidelines of the Guangdong General Hospital for Laboratory Animal Studies.

\section{Animal Model}

Fifteen pregnant goats with gestational age between 120 and 140 days were randomly and equally divided into a control group (CG), a fetal bypass group (FB), and a fetal bypass with pyrrolidine dithiocarbamate (PDTC) group (FP). PDTC, a specific antagonist of NF-KB, was given intravenously in a dose of $300 \mathrm{mg}$ after fetal sternotomy to each animal in the FP group. Surgical preparation and fetal cardiac bypass technique were carried out as previously described. ${ }^{15}$ The CG group underwent fetal sternotomy and cannulation without fetal cardiac bypass. The other groups underwent fetal cardiac bypass for 30 minutes. Hemodynamic data points of the maternal and fetal goats were continuously recorded using a model 64S monitor (Hewlett-Packard Company, Palo Alto, Calif).

\section{Fetal Cardiac Bypass}

As previously described, ${ }^{15}$ a fetal median sternotomy was performed and the pericardium was opened. Fetal cardiac bypass was achieved by cannulation of the main pulmonary artery using an $8 \mathrm{~F}$ DLP arterial cannula and the right atrium with a $12 \mathrm{~F}$ angled-tipped venous cannula (Medtronic, Inc, Minneapolis, Minn). A normothermic and nonpulsatile pump system, consisting of a centrifugal pump (Medtronic, BP50), the placenta, and a minimal heat exchanger (Derun Medical Instrument Co, Guangdong, China) was primed with $150 \mathrm{~mL}$ adult goat blood and $50 \mathrm{~mL}$ lactated Ringer solution containing $10 \mathrm{mg}$ heparin. No steroid was used in the pump prime. The flow rate was maintained at least $250 \mathrm{~mL} \cdot \mathrm{kg}^{-1} \cdot \mathrm{min}^{-1}$ for 30 minutes in both groups FB and FP before bypass was terminated, and all the animals were monitored for 2 hours before they were humanely killed for autopsy and measurement of fetal weight.

\section{Placental Vascular Resistance}

Umbilical mean arterial pressure and flow rate were measured using a 22-gauge indwelling catheter in the lumen of an umbilical artery and an ultrasound blood flowmeter (Transonic Systems, Inc, Ithaca, NY) in the same artery. Placental vascular resistance was calculated as the ratio of umbilical mean arterial pressure over flow rate.

\section{Blood Sample Regimen}

Fetal blood samples from the umbilical vein were collected for blood gas analysis with a GEM Premier 3000 blood gas analyzer (Instrumentation Laboratory, Bedford, Mass) immediately before cardiac bypass (T0), at 30 minutes into and immediately before termination of cardiac bypass (T1), and 1 and 2 hours after bypass (T2 and T3). All the fetal blood samples for inflammatory mediator assays were collected in a similar fashion and time points and placed immediately in test tubes containing ethylenediaminetetraacetic acid on ice, centrifuged at $3000 \mathrm{rpm}$ for 15 minutes in a cooled centrifuge, and the supernatants were then frozen at $-70^{\circ} \mathrm{C}$.

\section{Assay of Inflammatory Mediator}

Plasma NO level was measured by a chemical fluorescence technique (Nanjing Juli Biological Technical Co, Nanjing, China). Radioimmunoassays (Beijing Ke Mei Dong Ya Biological Technical Co, Beijing, China) were used to assay fetal plasma levels of ET-1 as well as 6-keto-prostaglandin $\mathrm{F} 1 \alpha(6-\mathrm{K})$ and thromboxane $\mathrm{B}_{2}$ (TXB2), metabolic substrates of prostacyclin and TXA2. Fetal plasma levels of IL-6 and TNF- $\alpha$ were assayed by enzyme-linked immunosorbent assay (Guangzhou Weijia Biological Technical Co, Guangzhou, China).

\section{Tissue Collection}

Placental samples of approximately $200 \mathrm{mg}$ from the fetal side were harvested after sternotomy at time point $\mathrm{T} 0$ and at other corresponding time points T1, T2, and T3. Samples were immediately frozen in liquid nitrogen.

\section{Real-Time Polymer Chain Reaction Analysis}

Total RNA was extracted from fetal placental tissues collected at T0 and T3 with Trizol reagent (Invitrogen, Carlsbad, Calif) and the concentrations were spectrophotometrically determined. Real-time quantitative reverse transcriptase polymer chain reaction analysis was performed with an ABI 7700 Sequence Detection System using TaqMan chemistry (Applied Biosystems, San Jose, Calif). Primers for IL-6 were 5'-GCTGCTCC TGGTGATGACTTC- $3^{\prime}$ and $5^{\prime}$-GGTGGTGTCATTTTTGAAATCTTCT $-3^{\prime}$. The amplicon was detected using the bifunctional fluorogenic probe $5^{\prime}$-FAM-CTTTCCCTACCCCGGGTCCC- TAMRA- ${ }^{\prime}$. Primers for TNF- $\alpha$ were $5^{\prime}$-TTCAACAGGCCTCTGGTTCAG- $3^{\prime}$ and $5^{\prime}$-TGGGCTACCGGC TTGTTACT- $3^{\prime}$, and fluorogenic probe was $5^{\prime}$-FAM-CACTCAGGTCATC TTCTCAAGCCTC-TAMRA- $3^{\prime}$. All reactions were performed in duplicate. Final results are absolute copy number per $1 \mathrm{mg}$ total RNA.

\section{Electrophoretic Mobility Shift Assay (EMSA)}

NF-KB binding was determined by EMSA. Nuclear protein from all the fetal placental tissue samples were extracted with NE-PER nuclear and cytoplasmic extraction reagents (Pierce Protein Research Products, division of Thermo Fisher Scientific, Rockford, Ill) according to the manufacturer's instructions. The probe oligonucleotide was 22-bp, double-stranded (5'-GCCTGGGAAAGTCCCCTCAACT-3'), and end-labeled with biotin (Yinjun Biological Technical Co, Shanghai, China). DNA-binding reactions were loaded into a $6 \%$ polyacrylamide gel, separated at $100 \mathrm{~V}$ for 30 minutes, and transferred at $100 \mathrm{~V}$ for 30 minutes to a positively charged 
nylon membrane. Transferred DNA was immediately cross-linked to the membrane on an ultraviolet transilluminator equipped with 312-nm bulbs and detected using horseradish peroxidase-conjugated streptavidin (LightShift Chemiluminescent EMSA kit) according to the manufacturer's instructions.

\section{Statistical Analysis}

IL-6 and TNF- $\alpha$ mRNA levels in each group were compared by the Student $t$ test. Intergroup comparisons were performed by repeated-measures analysis of variance. Intragroup comparisons were performed by 1-way analysis of variance. Statistical analysis was performed with the SPSS 13.0 software package (SPSS, Inc, Chicago, Ill). The data were expressed as means \pm standard deviation.

\section{RESULTS}

\section{Fetal Hemodynamics, Placental Vascular Resistance,} and Placental Gas Exchange

The mean bypass flow rates in the FB group $(290 \pm 32$ $\left.\mathrm{mL} \cdot \mathrm{kg}^{-1} \cdot \mathrm{min}^{-1}\right)$ and FP group $\left(289 \pm 32 \mathrm{~mL} \cdot \mathrm{kg}^{-1}\right.$. $\mathrm{min}^{-1}$ ) were not significantly different. The mean dosage of PDTC in the FP group was $175 \pm 25 \mathrm{mg} / \mathrm{kg}$. As shown in Table 1, no significant difference in mean arterial pressure was detected among the 3 groups during the experiments. The heart rate was higher before bypass and returned to fetal physiologic level during and after bypass in the FP group. Overall, heart rates were not significantly different among the 3 groups.

Placental vascular resistance and blood gas values remained within normal physiologic range in the $\mathrm{CG}$ group. Placental vascular resistance increased markedly in the FB and FP groups at T3 (Table 1). The partial pressure of carbon dioxide increased significantly, accompanied by a remarkable decline in $\mathrm{pH}$ and base excess values in the $\mathrm{FB}$ and FP groups. However, no significant difference was detected in oxygen tension among the 3 groups.

\section{Inflammatory Mediators}

As shown in Figure 1, the plasma levels of NO gradually increased in all 3 groups, and differences among the groups were significant $(P=.016)$. The plasma level of NO in the FB group was higher than that in the CG group; however, no difference was observed between either the FB and FP groups or the FP and CG groups. The difference among the 3 groups in the plasma level of ET-1 was remarked $(P=.048)$. Although the plasma ET-1 did not show any

TABLE 1. Fetal hemodynamics, placental vascular resistance, and placental gas exchange

\begin{tabular}{|c|c|c|c|c|c|}
\hline Group & T0 & T1 & $\mathbf{T 2}$ & $\mathbf{T 3}$ & $P$ value \\
\hline \multicolumn{6}{|l|}{ MAP (mm Hg) } \\
\hline CG & $51.6 \pm 11.0$ & $47.4 \pm 9.0$ & $46.6 \pm 13.4$ & $46.2 \pm 11.4$ & .867 \\
\hline FB & $55.6 \pm 9.8$ & $58.0 \pm 7.8$ & $51.0 \pm 1.4$ & $47.0 \pm 6.6$ & .111 \\
\hline $\mathrm{FP}$ & $58.6 \pm 5.8$ & $63.8 \pm 11.9$ & $56.4 \pm 7.1$ & $52.2 \pm 6.3$ & .195 \\
\hline \multicolumn{6}{|l|}{ HR (beats/min) } \\
\hline CG & $188 \pm 28$ & $173 \pm 39$ & $177 \pm 29$ & $173 \pm 25$ & .829 \\
\hline FB & $202 \pm 25$ & $142 \pm 20$ & $154 \pm 22$ & $150 \pm 66$ & .096 \\
\hline FP & $195 \pm 32$ & $152 \pm 19^{*}$ & $175 \pm 12$ & $159 \pm 10^{*}$ & .019 \\
\hline \multicolumn{6}{|c|}{$\operatorname{PVR}\left(\mathrm{mm} \mathrm{Hg} \cdot \mathrm{mL}^{-1} \cdot \mathrm{kg}^{-1} \cdot \mathrm{min}^{-1}\right)$} \\
\hline CG & $0.25 \pm 0.06$ & $0.28 \pm 0.05$ & $0.28 \pm 0.08$ & $0.29 \pm 0.04$ & .663 \\
\hline FB & $0.35 \pm 0.08$ & $0.64 \pm 0.22$ & $0.82 \pm 0.47$ & $1.31 \pm 0.59^{*}$ & .010 \\
\hline FP & $0.33 \pm 0.06$ & $0.64 \pm 0.36$ & $0.60 \pm 0.23$ & $0.98 \pm 0.36^{*}$ & .020 \\
\hline \multicolumn{6}{|l|}{$\mathrm{pH}$} \\
\hline CG & $7.31 \pm 0.06$ & $7.35 \pm 0.05$ & $7.31 \pm 0.06$ & $7.32 \pm 0.03$ & .429 \\
\hline $\mathrm{FB}$ & $7.35 \pm 0.08$ & $7.27 \pm 0.06$ & $7.24 \pm 0.05$ & $7.04 \pm 0.14 *$ & .010 \\
\hline FP & $7.35 \pm 0.06$ & $7.36 \pm 0.05$ & $7.22 \pm 0.04$ & $7.13 \pm 0.08^{*}$ & .000 \\
\hline \multicolumn{6}{|l|}{$\mathrm{PCO}_{2}(\mathrm{~mm} \mathrm{Hg})$} \\
\hline CG & $40.6 \pm 2.2$ & $42.0 \pm 5.3$ & $41.8 \pm 6.2$ & $43.6 \pm 3.8$ & .788 \\
\hline FB & $38.2 \pm 7.2$ & $46.8 \pm 5.9$ & $50.0 \pm 11.2^{*}$ & $64.8 \pm 9.5^{*}$ & .010 \\
\hline FP & $39.6 \pm 5.5$ & $44.8 \pm 5.0$ & $49.8 \pm 1.9^{*}$ & $60.4 \pm 4.8^{*}$ & .000 \\
\hline \multicolumn{6}{|l|}{$\mathrm{Po}_{2}(\mathrm{~mm} \mathrm{Hg})$} \\
\hline CG & $28.8 \pm 3.0$ & $30.0 \pm 4.4$ & $29.2 \pm 3.1$ & $28.0 \pm 1.6$ & .784 \\
\hline FB & $29.4 \pm 4.6$ & $26.0 \pm 4.2$ & $25.8 \pm 4.9$ & $26.4 \pm 5.3$ & .607 \\
\hline FP & $27.8 \pm 6.4$ & $28.8 \pm 2.6$ & $29.8 \pm 6.1$ & $21.4 \pm 6.4$ & .119 \\
\hline \multicolumn{6}{|l|}{ Base excess $(\mathrm{mmol} / \mathrm{L})$} \\
\hline CG & $-3.8 \pm 2.6$ & $-3.4 \pm 2.4$ & $-2.9 \pm 2.0$ & $-4.1 \pm 3.2$ & .906 \\
\hline FB & $-3.4 \pm 4.4$ & $-7.4 \pm 4.7$ & $-5.2 \pm 5.2$ & $-14.2 \pm 2.9^{*}$ & .007 \\
\hline FP & $-5.4 \pm 3.5$ & $-6.8 \pm 3.1$ & $-4.8 \pm 3.0$ & $-11.9 \pm 1.6^{*}$ & .006 \\
\hline
\end{tabular}

$T O$, Immediately before cardiac bypass; $T 1$, at 30 minutes into and immediately before termination of cardiac bypass; $T 2,1$ hour after bypass; $T 3,2$ hours after bypass; $M A P$, Mean arterial blood pressure; $H R$, heart rate; $P V R$, placental vascular resistance; $P \mathrm{Co}_{2}$, partial pressure of carbon dioxide; $P \mathrm{O}_{2}$, oxygen tension; $C G$, control group; $F B$, fetal cardiac bypass group; FP, fetal cardiac bypass with pyrrolidine dithiocarbamate group. ${ }^{*} P<.05$ compares in-group versus $\mathrm{T} 0$. 

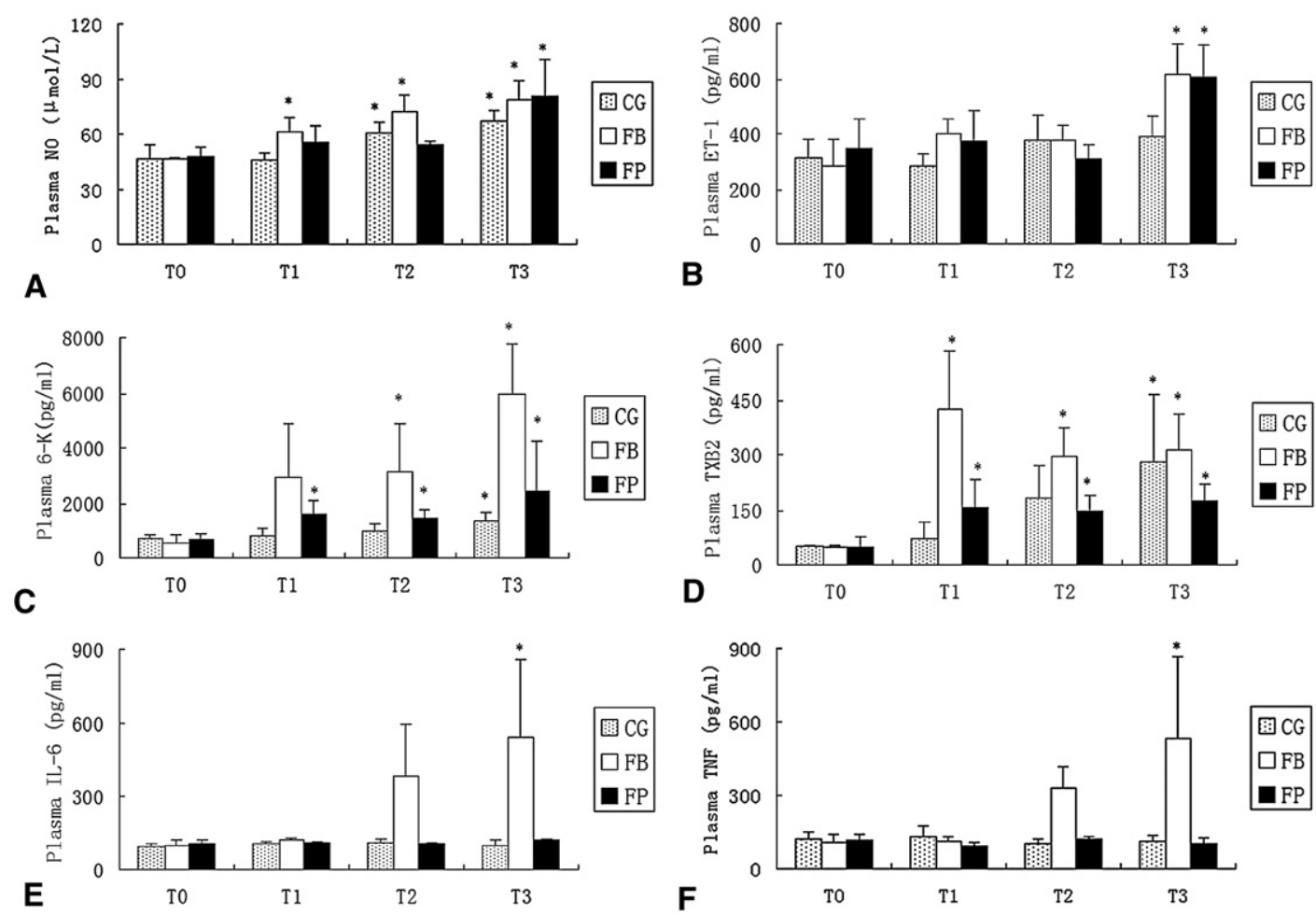

FIGURE 1. Fetal plasma inflammatory mediators: A, Changes in fetal plasma nitric oxide (NO). B, Changes in fetal plasma endothelin-1 (ET-1). $\mathrm{C}$, Changes in fetal plasma 6-keto-prostaglandin $\mathrm{F} 1 \alpha(6-K)$. D, Changes in fetal plasma thromboxane $\mathrm{B}_{2}(T X B 2)$. E, Changes in fetal plasma interleukin 6 (IL-6). F, Changes in fetal plasma tumor necrosis factor- $\alpha(T N F)$. $* P<.05$ comparison within groups versus T0. $C G$, Control group; $F B$, fetal bypass group; $F P$, pyrrolidine dithiocarbamate group; $T 0$, immediately before cardiac bypass; $T 1$, at 30 minutes into and immediately before termination of cardiac bypass; $T 2,1$ hour after bypass; $T 3,2$ hours after bypass.

obvious change in the CG group, it showed remarkable elevations in the FB and FP groups at T3 without any significant difference between them. Significant differences in plasma 6-K and TXB2 levels were seen among groups (6$\mathrm{K}, P=.010$; TXB2, $P=.015)$, although these clearly increased for all 3 groups. Plasma levels of $6-\mathrm{K}$ and TXB2 were the highest in the FB group, with no significant difference between the FP and CG groups. The difference among the 3 groups in plasma IL- 6 and TNF- $\alpha$ levels was marked (IL-6, $P=.012$; TNF- $\alpha, P=.016$ ), with higher levels in the FB group compared with the FP and CG groups. No significant changes were seen between the FP and CG groups. Likewise, levels of IL- 6 and TNF- $\alpha$ mRNA in the placental tissue were markedly elevated at T3 in the FB group compared with the CG and FP groups, but no significant difference was detected between the CG and FP groups (Table 2).

\section{EMSA for NF-KB}

Band shifts for the CG group showed no changes throughout the experiment. Significant changes were seen at $\mathrm{T} 2$ and $\mathrm{T} 3$ compared with $\mathrm{T} 0$ in the FB group. The changes in the FP group were significant at $\mathrm{T} 2$ but not at T3. EMSA results for T3 showed NF-KB was activated in FB group, but not in the FP group (Figure 2).

\section{DISCUSSION}

Our study showed that several plasma inflammatory mediators with various vasoactive properties were released after fetal cardiac bypass with subsequent placental vasoconstriction and the accompanying fetal hypercarbia and acidosis. The plasma levels of ET-1, a strong vasoconstrictive agent, increased significantly after fetal cardiac bypass with subsequent increase in placental vascular resistance in

TABLE 2. Levels of IL-6 and TNF- $\alpha$ mRNA

\begin{tabular}{lccc}
\hline \multicolumn{1}{c}{ Group } & T0 & T3 & $P$ value \\
\hline IL-6 mRNA $\left(10^{6}\right.$ copies/ $\mu \mathrm{g}$ & & & \\
$\quad$ total RNA $)$ & & & \\
CG & $1.10 \pm 0.55$ & $1.40 \pm 0.48$ & .270 \\
FB & $1.71 \pm 0.65$ & $3.24 \pm 1.38$ & .027 \\
FP & $1.10 \pm 0.57$ & $1.55 \pm 0.95$ & .235 \\
TNF- $\alpha$ mRNA $\left(10^{6}\right.$ copies/ $\mu \mathrm{g}$ & & & \\
$\quad$ total RNA) & & & \\
CG & $0.59 \pm 0.29$ & $0.76 \pm 0.37$ & .261 \\
FB & $0.90 \pm 0.40$ & $2.18 \pm 0.94$ & .044 \\
FP & $0.73 \pm 0.29$ & $0.81 \pm 0.58$ & .714 \\
\hline
\end{tabular}

$T 0$, Immediately before cardiac bypass; $T 3,2$ hours after bypass; $I L-6$, interleukin 6 ; $T N F-\alpha$, tumor necrosis factor- $\alpha$; $m R N A$, messenger RNA; $C G$, control group; $F B$, fetal cardiac bypass group; $F P$, fetal cardiac bypass with pyrrolidine dithiocarbamate group. 


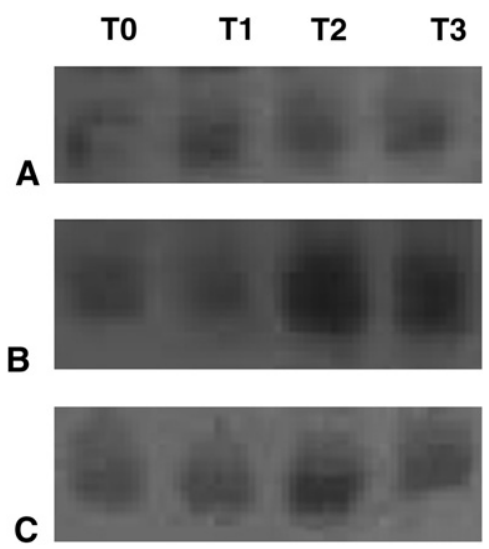

FIGURE 2. Electrophoretic mobility shift assay (EMSA) for nuclear factor kappa-B (NF-KB) band shift for (A) group CG at 4 time points, (B) group FB at 4 time points, and (C) group FP at 4 time points. $C G$, Control group; $F B$, fetal bypass group; $F P$, pyrrolidine dithiocarbamate group; $T 0$, immediately before cardiac bypass; $T 1$, at 30 minutes into and immediately before termination of cardiac bypass; T2, 1 hour after bypass; T3, 2 hours after bypass.

our study. A high level of NO, a known potent vasodilator, was observed after manipulation in utero and did not seem to have any effect on ET-1 at all. The plasma levels of 6-K and TXB2, the metabolic substrates of prostacyclin and TXA2, respectively, increased during and after fetal cardiac bypass. Prostacyclin and TXA2 had contrary vasoactive properties, but the combined effects on the placental vascular tone were vasoconstriction. The plasma levels of IL-6 and TNF- $\alpha$, which were detrimental to placental functions as previously reported, ${ }^{3}$ were also released significantly after fetal cardiac bypass in our study.

NF-KB is a major transcription factor involved in the regulation of inflammatory response, initiating transcription of genes for cyclooxygenase, NOS, ET-1, and some inflammatory mediators, such as TNF- $\alpha$ and IL- 6 . The EMSA results in our study showed NF-KB in the placental tissues was persistently activated after fetal cardiac bypass in the FB group. The transcription levels of IL- 6 and TNF- $\alpha$ mRNA in the placental tissues were also markedly increased in the FB group. These results and changes of inflammatory mediators suggested that NF-KB might be involved in the inflammatory response induced by fetal cardiac bypass.

PDTC is a relatively selective NF-KB inhibitor by a mechanism that prevents degradation of inhibitor kappaB. ${ }^{16}$ We did not explore the dose-response relationship of PDTC and instead opted to use $300 \mathrm{mg}$ in the FP group based on a previous study ${ }^{17}$ since the weights of the fetal goats cannot be measured before the onset of the experiments and the consideration that the bioavailability of PDTC might be influenced by the placental exchange and maternal metabolism. With the addition of PDTC to the FP group, the amount of activated NF-KB was noticeably reduced after fetal cardiac bypass compared with the FB group. The polymer chain reaction assay revealed that the transcription levels of IL- 6 and TNF- $\alpha$ mRNA in the placental tissues remained relatively unchanged in the FP group but significantly increased in the FB group. We also observed that other than NO and ET-1, all the inflammatory mediators assayed (6-K, TXB2, IL-6, and TNF- $\alpha$ ) were to various extents suppressed in the FP group, and yet placental dysfunction with hypercarbia and acidosis were observed in this group, not unlike the FB group, which did not receive PDTC.

Reddy and colleagues ${ }^{4}$ reported that the basal endothelial regulatory mechanisms of placental vascular tone were deranged and ET-1 played the crucial roles in the placental dysfunction after fetal cardiac bypass. PDTC suppressed the activation of NF-KB but had no effects on ET-1 in our study. It was reported that PDTC might reduce the expression of inducible NOS mRNA via the inhibition of activation of NF-KB. ${ }^{18}$ However, our study showed that PDTC did not interfere with the release of $\mathrm{NO}$ in the placenta after fetal cardiac bypass, and the coexisting high levels of NO and ET-1 resulted in increased placental vascular tone after fetal cardiac bypass, despite the fact that other inflammatory mediators including 6-K, TXB2, IL-6, and TNF- $\alpha$ were suppressed in the FP group. These results implicated that ET-1 might be the inflammatory mediator primarily responsible for the increased placental vascular resistance and dysfunction.

Recently, angiogenic imbalance is believed to lead to endothelial dysfunction, systemic vasoconstriction, and hypertension. Soluble fms-like tyrosine kinase 1 is an antiangiogenic factor against proangiogenic factor, such as vascular endothelial growth factor in placenta. ${ }^{19}$ Murphy and associate ${ }^{20}$ reported that ET-1 appears to play an important role in mediating the hypertension produced by chronic, excess soluble fms-like tyrosine kinase 1 in normal pregnant rats. Long-term treatment with an elective ET-1 receptor antagonist significantly reduced mean arterial pressure in soluble fms-like tyrosine kinase 1 hypertensive pregnant rats while having no effect on normal pregnant rats. This interaction between ET-1 and soluble fms-like tyrosine kinase 1 might provide new direction for future investigation in the field of fetal cardiac bypass and preservation of placental function.

In summary, our data suggested that fetal cardiac bypass activated NF-KB in placental tissue, promoted the placental inflammatory response, and affected placental function. However, pharmacologic inhibition of NF-KB with PDTC did not improve placental function. ET-1, among all the inflammatory mediators we assayed, might play a primary role in causing placental dysfunction.

\section{References}

1. Sabik JF, Assad RS, Hanley FL. Prostaglandin synthesis inhibition prevents placental dysfunction after fetal cardiac bypass. J Thorac Cardiovasc Surg. 1992 103:733-42. 
2. Sabik JF, Heinemann MK, Assad RS, Hanley FL. High-dose steroids prevent placental dysfunction after fetal cardiac bypass. J Thorac Cardiovasc Surg. 1994; 107:116-25.

3. Reddy VM, McElhinney DB, Rajasinghe HA, Rodriguez JL, Hanley FL. Cytokine response to fetal cardiac bypass. J Matern Fetal Invest. 1998;8:46-9.

4. Reddy VM, McElhinney DB, Rajasinghe HA, Liddicoat JR, HendricksMunoz K, Fineman JR, et al. Role of the endothelium in placental dysfunction after fetal cardiac bypass. J Thorac Cardiovasc Surg. 1999;117:343-51.

5. Parry AJ, Petrossian E, McElhinney DB, Reddy VM, Hanley FL. Neutrophil degranulation and complement activation during fetal cardiac bypass. Ann Thorac Surg. 2000;70:582-9.

6. Carotti A, Emma F, Picca S, Iannace E, Albanese SB, Grigioni M, et al. Inflammatory response to cardiac bypass in ewe fetuses: effects of steroid administration or continuous hemodiafiltration. J Thorac Cardiovasc Surg. 2003;126:1839-50.

7. Mak KK, Gude NM, Walters WA, Boura AL. Effects of vasoactive autacoids on the human umbilical-fetal placental vasculature. Br J Obstet Gynaecol. 1984;91: 99-106.

8. Sand A, Andersson E, Fried G. Nitric oxide donors mediate vasodilation in human placental arteries partly through a direct effect on potassium channels. Placenta. 2006;27:181-90.

9. McCarthy AL, Woolfson RG, Evans BJ, Davies DR, Raju SK, Poston L. Functional characteristics of small placental arteries. Am J Obstet Gynecol. 1994; 170:945-51.

10. Kingdom JCP, Burrell SJ, Kaufmann P. Pathology and clinical implications of abnormal umbilical artery Doppler waveforms. Ultrasound Obstet Gynecol. 1997; 9:271-86.
11. Hayden MS, Ghosh S. Shared principles in NF-kappaB signaling. Cell. 2008; 132:344-62.

12. Böhrer H, Nawroth PP. Nuclear factor $\mathrm{kB}$ - a new therapeutic approach? Intensive Care Med. 1998;24:1129-30.

13. Chen F, Castranova V, Shi X. New insights into the role of nuclear factor-kB, a ubiquitous transcription factor in the initiation of diseases. Clin Chem. 1999; 45:7-17.

14. Lindström TM, Bennett PR. The role of nuclear factor kappa B in human labour. Reproduction. 2005;130:569-81.

15. Zhou C, Zhuang J, Zhang X, Zhang J. Changes in atrial natriuretic peptide levels during cardiac bypass in the fetal goat. Artificial Organs. 2008;32:956-61.

16. Liu SF, Ye X, Malik AB. Pyrrolidine dithiocarbamate prevents I-kB degradation and reduces microvascular injury induced by lipopolysaccharide in multiple organs. Mol Pharmacol. 1999;55:658-67.

17. Liu SF, Ye X, Malik AB. Inhibition of NF-KB activation by pyrrolidine dithiocarbamate prevents in vivo expression of proinflammatory genes. Circulation. 1999;100:1330-7.

18. Liu SF, Ye X, Malik AB. In vivo inhibition of nuclear factor-kappa B activation prevents inducible nitric oxide synthase expression and systemic hypotension in a rat model of septic shock. J Immunol. 1997;159:3976-83.

19. Maynard SE, Venkatshea S, Thadhani R, Karumanchi SA. Soluble fms-like tyrosine kinase 1 and endothelial dysfunction in the pathogenesis of preeclampsia. Pediatr Res. 2005;57:1R-7.

20. Murphy SR, LaMarca BBD, Cockrell K, Granger JP. Role of endothelin in mediating soluble fms-like tyrosine kinase 1-induced hypertension in pregnant rats. Hypertension. 2010;55:394-8. 